Projets

de paysage

\section{Projets de paysage}

Revue scientifique sur la conception et l'aménagement de l'espace

$22 \mid 2020$

Forêt et paysage

\title{
La forêt linéaire
}

Un paysage en projet pour l'Eurométropole Likoto

The Linear Forest - A landscape in the Making for the Likoto Eurometropolis

Denis Delbaere

\section{Q OpenEdition}

1 Journals

Édition électronique

URL : http://journals.openedition.org/paysage/9032

DOI : 10.4000/paysage.9032

ISSN : 1969-6124

Éditeur :

École nationale supérieure du paysage de Versailles-Marseille, Institut national des sciences appliquées Centre Val de Loire - École de la nature et du paysage, École nationale supérieure d'architecture et de paysage de Bordeaux, École nationale supérieure d'architecture et de paysage de Lille, Agrocampus Angers

\section{Référence électronique}

Denis Delbaere, "La forêt linéaire », Projets de paysage [En ligne], 22 | 2020, mis en ligne le 21 juillet 2020, consulté le 24 juillet 2020. URL : http://journals.openedition.org/paysage/9032 ; DOI : https:// doi.org/10.4000/paysage. 9032

Ce document a été généré automatiquement le 24 juillet 2020.

Projets de paysage 


\title{
La forêt linéaire
}

\author{
Un paysage en projet pour l'Eurométropole Likoto \\ The Linear Forest - A landscape in the Making for the Likoto Eurometropolis
}

Denis Delbaere

1 La matière d'un immense projet de paysage, porteur peut-être d'une conception renouvelée de l'espace forestier, est en train d'émerger des espaces les moins considérés et pourtant aussi les plus présents de nos environnements quotidiens, les dépendances, le plus souvent végétalisées, des grandes infrastructures de transport. C'est la thèse qui sera soutenue dans cet article, à partir des résultats d'un ensemble de recherches menées depuis près de dix années par une équipe pluridisciplinaire forte d'une quinzaine de membres ${ }^{1}$.

2 C'est bien à tort en effet qu'on réduirait les grandes infrastructures de transport aux plateformes, chaussées et biefs qui y portent le trafic ferroviaire, routier et fluvial. Le propre d'une infrastructure de transport est de rechercher le trajet le plus direct possible, en plan et en coupe, entre diverses destinations à relier, ce qui suppose un affranchissement relatif des niveaux de l'ouvrage par rapport à ceux du terrain dit naturel. Ces écarts sont rattrapés grâce à la construction de talus, dont les lignes de crête et les bases sont elles-mêmes précédées par des accotements plus ou moins larges. Depuis l'entre-deux-guerres, l'accroissement des moyens techniques de terrassement et des objectifs de vitesse et de volume porté, notamment grâce à l'invention du vérin hydraulique et à la révolution qu'il a représentée dans la technologie des engins de levage et de poussage, a eu pour effet d'amplifier l'emprise des talus et des accotements. Autoroutes, canaux à grand gabarit et lignes à grande vitesse ont éventré des plateaux et comblé des vallons au prix d'un accroissement considérable des surfaces de talus. La revue Espaces verts consacrait dès avril 1968 un dossier à ce sujet, sous la plume du paysagiste Alan Spake, qui a passé toute sa carrière dans les services successifs chargés du paysage au sein du ministère et des administrations des Transports. Ce dossier (Spake, 1968, p. 8-25) s'ouvrait sur la reproduction du plan de terrassement d'un échangeur autoroutier montrant, outre les modelés complexes qu'impose le raccordement des niveaux, à quel point la superficie que la partie utile de l'infrastructure occupe est désormais faible comparée à celle des talus. Parti 
d'Allemagne et d'Italie, le mouvement de construction d'un réseau dense de grandes infrastructures a trouvé en France ses mots d'ordre lorsque le Plan national d'aménagement du territoire, voulu par le très moderniste ministre de la Reconstruction Eugène Claudius-Petit, prescrivit la mise en place de schémas d'aménagement routier ambitieux, déclinés à l'échelle de chaque région, complétés et revus marginalement après leur première publication au début des années 1960, et pour l'essentiel mis en œuvre avec détermination par les services des Transports dans les décennies qui suivirent ${ }^{2}$.

Aujourd'hui, les talus des autoroutes, lignes de train régionales ou internationales et canaux à grand gabarit sont des éléments banals des paysages urbains et périurbains, alors qu'ils ne possèdent aucune fonctionnalité propre. Ces délaissés, résultat des calculs des ingénieurs, sont des espaces perdus, sans valeur économique (s'ils protègent les riverains des dangers du trafic, leurs constructeurs leur auraient volontiers substitué des murs de protection s'ils n'avaient été si coûteux) et que les paysagistes ont parfois été chargés d'investir d'une fonction esthétique au moyen de plantations variées. Une histoire de ces plantations montre qu'elles ont surtout permis aux gestionnaires des infrastructures de se dédouaner de la charge financière que représenterait l'entretien de ces vastes surfaces: une fois planté, le talus a été en général laissé à lui-même, seule la bande de terrain la plus proche de la plateforme circulée étant régulièrement fauchée, et le reste évoluant lentement mais sûrement vers des formes incontrôlées de friches arborées. La succession végétale a joué ici avec une grande efficacité, et les paysagistes ont fini par abandonner leurs premiers exercices de plantation encore très marqués par leur culture jardiniste, tentant d'assimiler ces talus à de longs jardins linéaires aux compositions chromatiques et aux volumes étudiés, au profit de masses de taillis alternant cinétiquement avec de simples prairies de fauche. Ils leur ont ensuite souvent préféré la plantation de simples bosquets denses laissés à eux-mêmes, dont les préconisations de gestion, notamment par éclaircissement, ne furent jamais appliquées. 
Figure 1. Jean-Claude Saint-Maurice (paysagiste), étude des plantations pour l'autoroute du Nord

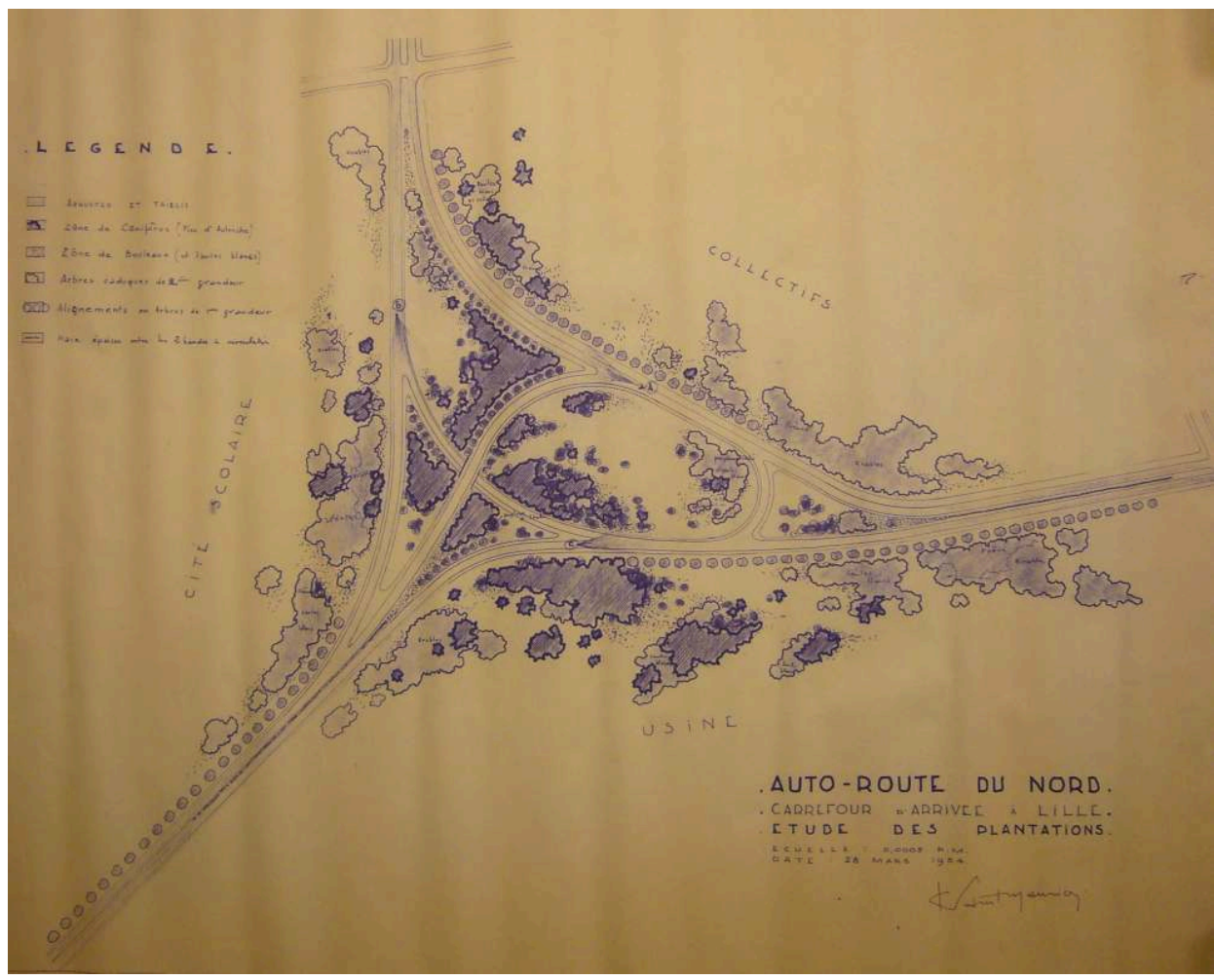

Source : Institut français d'architecture, fonds Leveau, nº 149, boîte $n^{\circ} 8$.

Figure 2. Plantations de la route départementale 700 au Sud de Roubaix en 2012

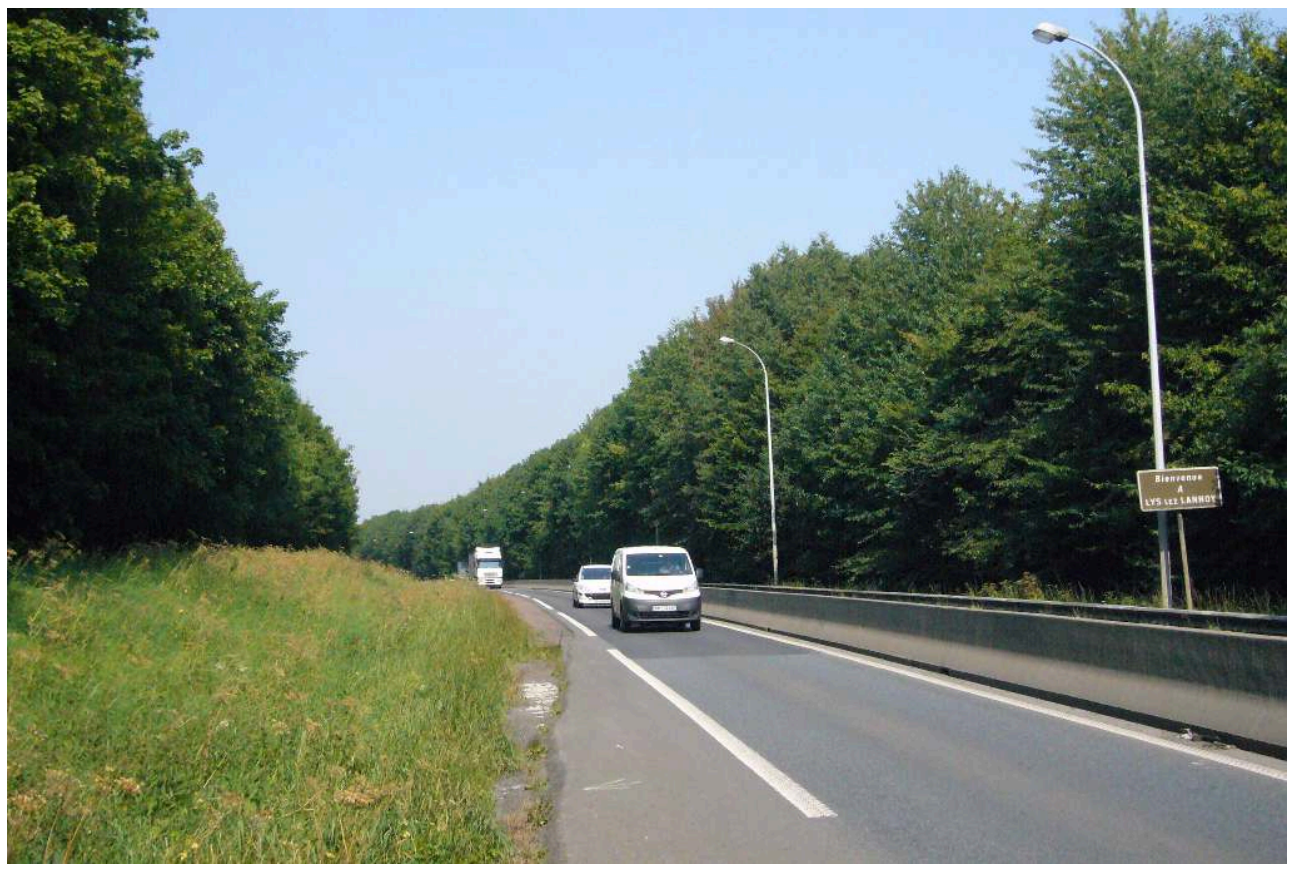

Source : Denis Delbaere.

4 Plantés pour la plupart entre 1970 et 1990, ces taillis ont effectivement permis à leurs gestionnaires de réaliser d'importantes économies tant qu'il s'agissait de les laisser pousser. Mais passées les premières décennies de croissance, les arbres ne trouvent 
plus assez d'espace pour se développer et ne parviennent à accéder à la lumière qu'en étirant leurs troncs le plus vite possible vers le ciel. Ces perches étiolées, fragiles et cassantes s'appuient les unes sur les autres et les coups de vent peuvent y jouer comme sur des champs de maïs. Les arbres menacent de tomber sur les plateformes circulées et d'y provoquer des accidents graves. De nombreux retards de trains en gare sont dus à des problèmes de ce type car une simple branche charpentière que le vent aurait poussée sur les rails provoque l'arrêt du trafic et appelle une intervention chronophage des services d'entretien ${ }^{3}$. La chute des arbres a également pour effet d'arracher une partie du talus et de déstabiliser ses parties les plus élevées, accroissant encore le risque de verse. Il semble bien dans ces conditions qu'après avoir été source d'économie, la plantation de ces talus devienne désormais source de dépenses, parfois considérables lorsqu'il faut défricher de longs linéaires et les convertir en surfaces de pelouse dont la fauche, annuelle ou bisannuelle, mobilise des équipes nombreuses, représente donc un coût salarial important dans un contexte de réduction budgétaire généralisée, et par ailleurs perturbe le trafic lorsque les interventions d'élagage, de défrichage ou de fauche se font depuis des plateformes ou chaussées qu'il faut neutraliser partiellement ou totalement ${ }^{4}$.

5 Mais ces problèmes de gestion, en bouleversant les équilibres et les habitudes, ouvrent la perspective d'un projet de paysage dont les conditions et les aspects ont été analysés dans le cas de l'Eurométropole Likoto (Lille-Kortrijk-Tournai). Les marges infrastructurelles de ce territoire ont fait l'objet d'explorations de terrain et de relevés dont l'objectif initial était d'évaluer dans quelle mesure ces espaces intéressent la conservation de la biodiversité en milieu urbain et périurbain. Deux programmes de recherche ${ }^{5}$ ont permis le financement et l'encadrement scientifique d'une démarche qui se poursuit aujourd'hui sous la conduite de l'auteur du présent article. Les résultats actuellement obtenus auraient sans doute pu l'être dans d'autres contextes territoriaux, et l'Eurométropole Likoto n'a servi de premier terrain d'investigation qu'en raison de l'ancrage géographique de l'équipe de recherche, de part et d'autre de la frontière franco-belge, et parce que cette Eurométropole est particulièrement riche en grandes infrastructures, avec ses $650 \mathrm{~km}$ d'autoroutes, voies ferrées et canaux en bordure du grand carrefour du Nord-Ouest européen.

\section{Derrière les logiques de contention, la stabilisation des boisements linéaires comme composante du paysage ordinaire}

6 Fondée initialement sur une démarche d'inventaire biologique et paysager, la recherche s'est très vite intéressée aux conditions de gestion de ces talus et accotements. La diversité biologique, évaluée dans ses dimensions floristiques et avifaunistiques, d'abord par une démarche d'inventaire exhaustive menée sur 25 placettes, puis en mobilisant une démarche de « diagnostic flash» (Lemoine, 2018) sur un linéaire continu de $60 \mathrm{~km}$, apparaît fortement impactée par les modes de gestion et une série d'entretiens a été réalisée auprès des services concernés (Métropole européenne de Lille, Infrabel, conseil départemental du Nord, direction interdépartementale des Routes Nord, service des Infrastructures wallonnes). Ces échanges avec les gestionnaires d'infrastructures mettent en évidence une logique 
globale de contention de la forêt linéaire, même si cette logique suit des chemins différents.

7 La Société nationale des chemins de fer (SNCF) est la société d'exploitation des infrastructures qui mène la politique la plus drastique du point de vue de la contention des boisements qui se sont formés sur les talus dont elle a la charge, et qui atteignent souvent des superficies considérables dans le cas des lignes à grande vitesse. Ces dernières sont apparues en effet tardivement dans le concert des infrastructures de transport métropolitaines, et ont dû composer avec un réseau déjà dense qu'il a fallu franchir en de nombreux points, générant des talus non seulement très nombreux mais aussi très longs car les rampes des voies ferrées doivent respecter une inclinaison particulièrement faible. La SNCF gère des centaines de kilomètres de talus souvent couverts par une sylve épaisse formée depuis une trentaine d'années. Les travaux de contention de cette sylve ont suivi essentiellement deux principes. Le premier consiste à appliquer le long des voies et sur une bande d'épaisseur variable de part et d'autre des traitements herbicides parfois administrés depuis des wagons spécialement affrétés ${ }^{6}$. La flore ligneuse est ainsi éliminée et sa repousse est très limitée, ce qui a favorisé l'implantation d'espèces herbacées, notamment thermophiles, qui diversifient considérablement le cortège floristique des voies ferrées et assurent parfois le maintien de plantes de milieu sec propres à la flore régionale des Hauts-de-France, mais attachées à des habitats naturels fortement raréfiés comme les pelouses calcaires. Le second principe consiste à mener des chantiers de défrichage complets sur une bande d'environ 15 mètres de largeur de chaque côté de la plateforme roulante. Les arbres ne sont ainsi maintenus que sur les hauts ou les bas de talus en sorte que, même en se couchant, leurs ramures ne risqueraient pas d'atteindre les voies. Ces travaux très fastidieux ont fait l'objet d'un contrat de gestion avec les services de l'office national des forêts (ONF), qui dispose d'une solide expérience en la matière et propose par ailleurs une expertise sur les arbres à conserver éventuellement dans le cadre de ces chantiers ${ }^{7}$. La paysagiste de l'ONF en charge de ces chantiers 8 intervient régulièrement pour identifier les sujets les plus solides et les mieux ancrés, mais aussi ceux dont la présence qualifie le mieux le paysage, et dont la conservation est parfois obtenue. Les plantes ligneuses, rarement dessouchées, repoussent évidemment dès la fin des opérations et forment en quelques mois de vastes surfaces de fourrés que la SNCF souhaite maintenir sous la barre des 2 mètres, au prix d'une reconduction régulière des travaux.

8 La direction interdépartementale des Routes (DIR), gestionnaire du réseau autoroutier, suit une logique analogue mais à des échelles différentes. Ici aussi une pelouse est maintenue le long des bandes d'arrêt d'urgence sur une largeur de 1,6 mètre. Le dégagement est cependant produit, depuis la loi relative à la transition énergétique, par la croissance verte du 17 août 2015 interdisant l'emploi de produits phytosanitaires sur les espaces publics, par des campagnes de fauche fastidieuses, en particulier lorsque le bas du talus est fermé par une glissière de sécurité dont les pieds doivent être contournés. Au-delà de cette bande, la DIR laisse désormais pousser les ligneux sans contrainte, les plus grands échangeurs évoluant vers un couvert arboré d'aulnes et de saules (blancs, marsault et cendrés), de frênes et d'érables sycomores, cernés de lisières de cornouillers, de sureaux, de prunelliers et de ronces'. Sur le versant wallon, comme on le verra plus loin, ces mesures ont été abandonnées au profit d'une approche plus radicale, mais qui subit actuellement de fortes mutations. 
9 La Métropole européenne de Lille (MEL) gère depuis quelques années les linéaires de canaux sur son territoire, en superposition de gestion avec les Voies navigables de France (VNF), mais aussi depuis le $1^{\text {er }}$ janvier 2017, dans le cadre de la loi NOTRe, les accotements des voiries auparavant gérés par le conseil général du Nord, et qui correspondent à des voies structurantes à l'échelle du territoire. À cela s'ajoutent les linéaires de voies secondaires dites communautaires, l'ensemble faisant de la MEL le gestionnaire de la plus grande partie des linéaires de Likoto. Les accotements des canaux font l'objet de plans de gestion précis, confiés à un service spécifique, et dont le cahier des charges évolue en fonction des situations ${ }^{10}$. C'est que la MEL a fait depuis plusieurs années de la reconquête et de la valorisation paysagère et environnementale du réseau de canaux l'un des axes principaux de sa politique en matière de cadre de vie. Seules les berges restent à la charge de VNF, qui y pratique une gestion particulièrement erratique. Le non-entretien des palplanches, tunages et enrochements a provoqué en quelques années l'installation et la croissance rapide d'aulnes, de saules et de frênes qui menacent finalement la solidité des tenues de berge. L'arrachage de cette ripisylve n'est réalisé que selon une périodicité lâche, parfois tous les 10 ans, tant le montage des dossiers de marché et des appels d'offres pour sélectionner les entreprises attributaires de ces travaux nécessite de longues phases d'instruction. On passe alors sans transition d'un paysage fluvial ourlé par les bandes boisées à des espaces complètement dégagés. La gestion des anciennes voiries départementales, quant à elle, a simplement reconduit les méthodes que le conseil général appliquait, et qui consistaient, selon une logique très proche de celle de la DIR, à préserver une bande d'accotement dégagée et à laisser les ligneux se développer à l'écart ${ }^{11}$.

Ces différences dans les pratiques de gestion sont finalement de degré plus que de nature. Toutes ont renoncé à éliminer totalement les boisements, source des difficultés, et se contentent d'en stopper le développement au-delà d'un certain seuil. Dans ces conditions, il est vraisemblable que ces boisements linéaires sont appelés à devenir des éléments pérennes du paysage eurométropolitain, d'autant que tous les gestionnaires insistent sur l'attachement que les usagers et les riverains de ces boisements manifestent à leur égard. Les premiers chantiers d'abattage engagés par le conseil général ou par VNF ont suscité des réactions immédiates des riverains, s'indignant auprès de leurs élus de la destruction de « leurs » arbres, et faisant pression sur eux, en général avec succès, pour obtenir l'arrêt rapide des travaux ${ }^{12}$.

\section{Le talus boisé comme espace public marginal}

11 Les réactions des riverains montrent que les boisements de bords d'infrastructures peuvent participer à ce qu'ils identifient comme une part intégrante de leur cadre de vie. De fait, les investigations menées sur les talus et leurs abords immédiats - sous forme d'entretiens libres avec les riverains et les usagers rencontrés, mais aussi à partir d'un questionnaire adressé en porte-à-porte aux habitants d'une série de lotissements le long de l'une des infrastructures étudiées - ont mis en évidence l'intensité de leur appropriation sociale, découverte parfois douloureuse pour les biologistes de l'équipe qui voyaient leurs pièges à insectes disparaître dans les jours qui suivaient leur mise en place, et devaient convenir que la biodiversité de ces espaces n'intéresse pas que les plantes et les animaux. L'abandon progressif et relatif des talus boisés a en fait laissé le champ libre à des pratiques sociales variées et souvent marginales, déjà décrites dans le 
cas grenoblois (Chelkhoff et al., 2016) dont nous avons dressé un état des lieux approximatif à partir du relevé d'indices d'usage et, quand cela était possible, d'entretiens avec les personnes rencontrées.

Les pratiques de promenade ont été régulièrement observées. Elles prennent place le plus souvent sur des pistes de bords de talus, plus ou moins entretenues, et servant tantôt d'accès aux ouvrages des services communautaires de l'écologie urbaine et de l'assainissement, nombreux autour de ces voies qui ont souvent perturbé le régime d'écoulement des eaux, tantôt de pistes cyclables ou encore de voies d'accès à des champs que le tracé autoroutier ou ferroviaire a coupé du reste des exploitations. Il est fréquent de croiser sur ces pistes, en lisière des boisements, des personnes seules ou des couples souvent accompagnés de leur chien, et qui trouvent ici un espace de promenade de proximité. Ces pistes sont également empruntées dans certains cas pour rallier plus rapidement un de ces équipements scolaires ou sportifs, collège, lycée, stade ou salle de sport, construits souvent sur les fonciers disponibles et vastes de ces périphéries, et dont l'accès par la route suit les circonvolutions et les détours compliqués qu'on connaît au système de voirie périurbain. Dans certains cas, un long itinéraire en voiture peut être remplacé par le raccourci offert par une piste caillouteuse entre lotissement, champs enclavés et talus boisés.

Des pratiques sportives sont régulièrement observées. Elles prennent essentiellement deux formes. La course à pied le long du talus et plus rarement à travers ses boisements est fréquente dès qu'on se trouve à proximité des tissus urbains. Les voies ferrées sont des supports appréciés, mais aussi certaines bordures d'autoroutes dont les pistes latérales permettent de boucler un parcours. La pratique du VTT est également très présente mais elle apparaît davantage dans les boucles des échangeurs, et donc sous la sylve. Nous avons relevé à plusieurs endroits des parcours aménagés et entretenus par leurs usagers. Des modelés de terrain, en butte et en creux, tassés comme des sculptures polies par le passage régulier des deux roues, confèrent à ces lieux au surgissement toujours inattendu une forte présence plastique. Des obstacles sont disposés à intervalles réguliers pour organiser les parcours et les slaloms. De petites passerelles sont construites pour enjamber les fossés trop profonds et étendre les parcours.

14 La dépose des ordures entre aussi largement dans le concert des pratiques sociales associées aux boisements infrastructurels. Il est fréquent de trouver, au fond des chemins finissant en impasse sur les talus, des tas d'ordures à proximité des lotissements : déchets de tonte, matériaux, briquaillons. Les lisières de ces boisements remplacent pour beaucoup de citoyens peu scrupuleux la déchetterie trop éloignée ou jugée trop coûteuse (sur le versant belge, les déchetteries sont payantes). Les gestionnaires des infrastructures déplorent largement ces pratiques qui les contraignent à des mesures de nettoyage fastidieuses et onéreuses ${ }^{13}$, mais parfois ils s'inscrivent aussi d'une certaine manière dans une même logique lorsqu'ils entreposent sous un pont des stocks de bordures, de pavés ou de sable afin de décongestionner des ateliers municipaux surchargés. 
Figure 3. Divers aspects de l'appropriation sociale des talus boisés

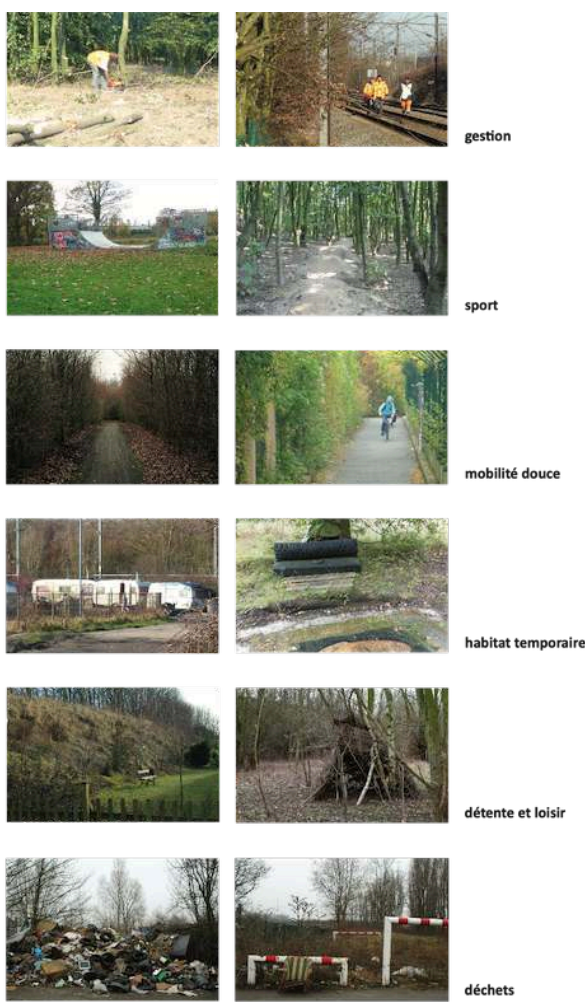

Voies de désenclavement agricole, potagers, promenades, accès aux ouvrages d'assainissement, aires de jeux improvisées, course à pied, raccourcis, graffitis et habitats temporaires...

Sources: Sabine Ehrmann et Denis Delbaere.

Les pratiques sexuelles laissent également sous les arbres de nombreux indices de leur présence: préservatifs abandonnés, voitures et monospaces aux vitres occultées, messages gravés dans l'écorce de certains arbres. Il en va de même pour la consommation et sans doute la vente de drogues, et pour la consommation d'alcool, laissant sur place de nombreux déchets, souvent autour de foyers sommairement aménagés entre des bordures de parpaings ébréchés ou de rondins de bois vert. Le nombre de canettes suggère des réunions sous les arbres, autour d'un feu, pour partager un repas de produits emballés arrosés de bière et de spiritueux bas de gamme.

Les talus boisés servent aussi de lieu d'habitat temporaire présentant des formes plus ou moins élaborées et indiquant une sédentarisation plus ou moins nette. Nous avons rencontré souvent de simples tentes, groupées par deux ou trois. Plus rarement il s'agit de cabanes dont la construction dénote une ingéniosité parfois remarquable. Ces lieux sont occupés en général de façon saisonnière, pendant les beaux jours ${ }^{14}$. Leur implantation induit des stratégies d'organisation du territoire parfois sophistiquées: pose de marques dans certains arbres pour indiquer les accès aux sentes menant, souvent, au bout d'un itinéraire long de plusieurs centaines de mètres, à une clairière habitée; pose de passerelles sommaires sur le fossé qui interdit l'accès au talus; percement de trous dans les grillages barrant l'accès ; mais aussi disposition de troncs d'arbres en travers de certains chemins trop visibles afin d'éloigner les curieux, installation de cordes entre les arbres pour faire sécher le linge, ou encore balayage méticuleux des abords de la tente ou de la cabane pour éviter de salir le sol à l'intérieur. L'habitat précaire prend aussi parfois la forme de campements implantés par les 
populations nomades ou marginalisées, notamment les Roms et plus rarement les Manouches ${ }^{15}$. Ces campements sont des ensembles élaborés et organisés, dont les abords sont parfois barrés par des obstacles et souvent parsemés de déjections humaines, présentant le double avantage de satisfaire l'interdit qui entoure la défécation chez ces populations en la repoussant loin du campement, et d'éloigner les curieux qui renoncent souvent à franchir cette barrière symbolique et explicite.

Ces pratiques sont donc de nature très variée mais ont en commun de tirer parti d'une même ressource, qui est formée précisément par les boisements. Dans le cas des Roms et des SDF, le bois devient une ressource matérielle et énergétique, utilisée pour les braseros et pour la construction. Même en l'absence d'outillage adapté, les habitants de ces bois linéaires parviennent à abattre de grands arbres, par un lent brulage de leurs collets, tandis qu'il n'est pas rare d'entendre en bordure des camps roms le travail de la hache et de la cognée. La densité de ces pratiques varie évidemment en fonction de la proximité de la ville ou de pôles d'attraction particuliers, comme les centres commerciaux dont les containers de poubelles sont particulièrement attractifs pour certains $\mathrm{SDF}^{16}$. Autant que nous ayons pu en juger cependant, rares sont les conflits entre ces pratiques pourtant parfois antagoniques. Les itinéraires de promenade restent en lisière, tandis que les habitats s'enfoncent dans la profondeur de la sylve. Certaines formes de symbiose peuvent même apparaître quand, par exemple, les habitants des talus ou les vététistes en quête de matériaux pour leurs circuits utilisent certains des déchets abandonnés par les riverains en lisière des talus.

De là à voir dans ces lieux une catégorie d'espace public urbain à part entière, il n'y a qu'un pas qu'il n'est pas absurde de franchir, si on veut bien considérer que les pratiques liées à ces refuges urbains sont profondément inscrites, qu'on le veuille ou non, dans l'ordre et les équilibres imparfaits de nos sociétés, et que l'environnement urbain de plus en plus policé des centres-villes et des périphéries résidentielles ne leur accorde plus aucune place légitime. La vie des talus boisés n'est pas alors sans évoquer celle des saltus médiévaux, ces espaces en marge des clairières féodales où une population variée d'ermites, de sorcières, de moines et de coupe-jarrets pouvait prendre place (Repoix et Richard, 2019). C'est le filigrane de la thèse que développe Claire Simon dans son riche documentaire sur les habitants et les usagers des marges du bois de Vincennes ${ }^{17}$, donnant ainsi un tour anthropologique à l'idée d'un retour paradoxal du sauvage dans le cœur des villes popularisé par les nouvelles de James Graham Ballard (2005) ou les romans de Philippe Vasset (2007).

\section{Les prémices d'une sylviculture urbaine}

19 Il serait sans doute exagéré d'affirmer que ces bois, plantés jadis pour occuper des espaces dénués de valeur fonctionnelle, sont d'ores et déjà devenus des composants à part entière du cadre de vie métropolitain, porteurs de leurs propres vertus et pris en compte de manière adaptée par ceux qui ont la charge de leur gestion. Mais il existe quelques indices d'une évolution de ces mesures de gestion tendant à valoriser ces espaces comme des ressources économiques. Déjà, les services gestionnaires déclarent utiliser le bois extrait lors des campagnes d'élagage ou de défrichage comme un revenu indirect pour les personnels qui y sont affectés. Par exemple, les grumes les plus grosses sont fréquemment distribuées aux employés de la DIR et de la MEL qui se réjouissent d'y trouver un complément de revenu pour le chauffage ${ }^{18}$. 
20 En Belgique, sur le versant wallon de l'Eurométropole, la valorisation économique du bois a atteint un stade supérieur depuis deux ans. Les infrastructures wallonnes présentent en effet des accotements particulièrement boisés, en raison notamment de la quantité et de la dimension des talus, omniprésents dans cette région au réseau routier et ferroviaire très dense, induisant des franchissements incessants, et dont les reliefs ondulants imposent aux tracés de nombreux passages en remblai ou en déblai. Plantés à partir des années 1960, ces talus ont laissé place à une véritable forêt linéaire poussant jusqu'au bord des voies et souvent entre elles sur de larges terre-pleins centraux. Depuis une dizaine d'années, les services routiers wallons ont engagé une politique de contention de ces boisements que nous avons relevée au début de cet article, mais en repoussant les arbres à une quinzaine de mètres des voies ${ }^{19}$. Ces mesures drastiques ont suscité les protestations de nombreux usagers et riverains, ainsi que la critique des services paysagistes de la direction des Infrastructures wallonnes, qui a notamment montré que la destruction de ces boisements, en laissant à nu les arbres qui en constituaient le cœur, a pour effet d'accroître leur fragilité au lieu de la réduire, car ces arbres ont perdu avec leurs voisins la béquille sur laquelle ils s'appuyaient, sans avoir à former un appareil racinaire solide. Ces arbres ramenés en lisière présentent donc un niveau de fragilité accru et d'autant plus préoccupant. Les services paysagistes ont donc proposé de substituer à la pratique de la coupe à blanc, générant une lisière droite et éloignée, l'application d'un profil de lisière étendue, laissée proche de la chaussée, et s'élevant de façon progressive en un large ourlet arbustif jusqu'à la strate arborée maintenue en partie haute ${ }^{20}$. L'étude puis la validation de ce nouveau protocole de gestion ont intégré les services de la Direction nationale de la forêt (DNF), de l'aménagement du territoire, et du Département des études des milieux naturels et agronomiques (DEMNA). Des personnalités comme Marc Dufresne, forestier de formation et spécialiste des services écosystémiques à l'Institut de Gembloux, ont joué un rôle important dans la conciliation entre les dimensions paysagères et environnementales du protocole, estimant que même si ses prescriptions peuvent être critiquées par certains aspects d'un point de vue environnemental, la situation vers laquelle elles font évoluer les boisements est structurellement positive et va globalement dans le sens d'un accroissement de la biodiversité ordinaire.

21 Dans ce contexte wallon, une telle mesure suppose évidemment une prise en compte assez fine des situations propres à chaque talus et à chaque infrastructure, et une complexification des chantiers de défrichage à laquelle les services routiers n'étaient bien sûr initialement pas favorables. Il a fallu une importante modification du statut juridique de ces boisements pour que le modèle préconisé soit finalement adopté et que sa mise en œuvre débute.

22 La gestion de ces boisements était en effet simple et entièrement laissée à l'appréciation des services gestionnaires. Cette situation a changé radicalement lorsque le ministre des Infrastructures et de l'Environnement, sur proposition de la Direction des aménagements paysagers, a accepté que les abords boisés soient gérés comme une forêt linéaire. Afin d'éviter de longues démarches administratives, les gestionnaires doivent désormais intégrer le protocole de gestion en lisière étalée prescrit par les paysagistes, et c'est bien sûr ce qui est fait de façon systématique.

Les premiers chantiers ont commencé depuis un an. Ils suivent les prescriptions produites au cas par cas par la petite équipe paysagiste de la direction des infrastructures ${ }^{21}$ en vue d'une évolution des talus vers le profil en lisière étalée. Par 
ailleurs, ces opérations dégagent un tel volume de bois que les entreprises chargées des travaux sont essentiellement rémunérées par sa récupération. La sylviculture urbaine terme qui sera discuté plus loin - qui s'invente ici permet aux gestionnaires de renouer avec leur objectif initial (rendre la maintenance de ces espaces inutiles, du point de vue de l'infrastructure, économiquement neutre) mais au bénéfice d'une diversification biologique et paysagère de talus boisés désormais assimilés à un élément à part entière du paysage à travers la figure tutélaire de la forêt. Ce mode de gestion émergent ouvre peut-être une voie médiane entre l'aveuglement normatif des méthodes actuellement dominantes et la précision de gestions plus différenciées. Sans doute plus vertueuses sur le plan environnemental et paysager, ces dernières se limitent en effet à quelques chantiers expérimentaux en raison de leur faible compatibilité avec l'organigramme contraint des services d'infrastructures.

Figure 4. Vue d'une portion récemment restructurée de l'autoroute de Wallonie

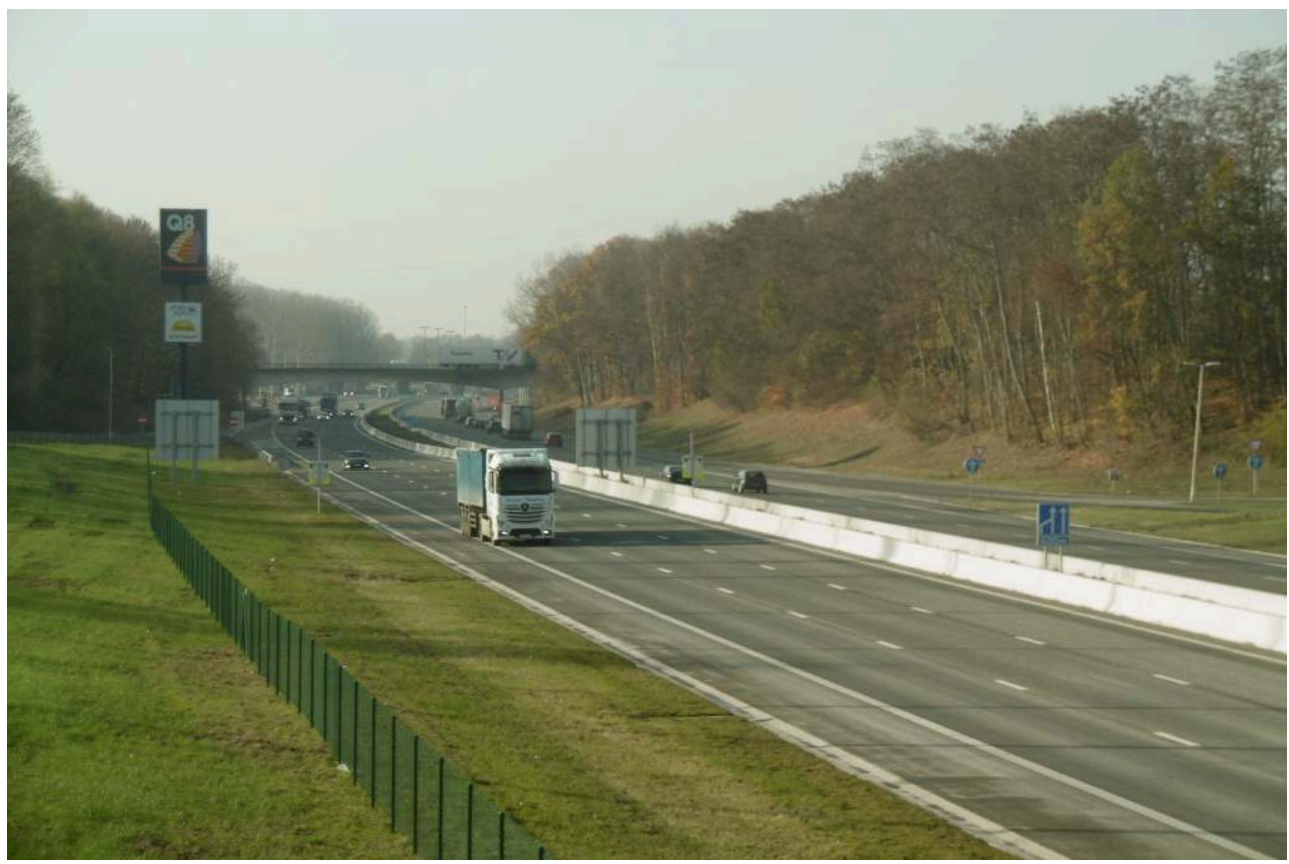

Sources : Sabine Ehrmann et Denis Delbaere. 
Figure 5. Extraits du Guide d'application $n^{\circ} 2$ « Bonnes pratiques et recommandations pour la gestion des abords boisés des bermes centrales et latérales situées sur les infrastructures routières régionales »

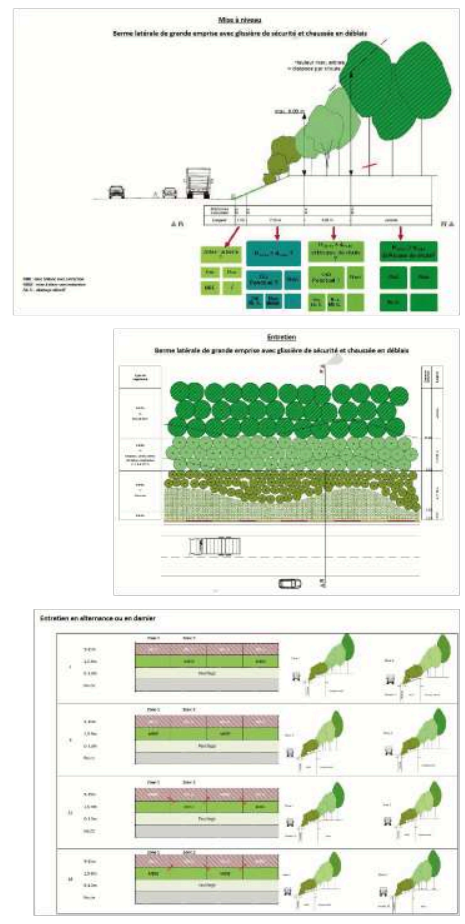

Ce guide est attaché à la circulaire ministérielle relative à la gestion des espaces paysagers présents sur le domaine des infrastructures régionales wallonnes, 2019.

\section{La forêt linéaire comme métaprojet de paysage}

La transfiguration paysagère des talus boisés en forêt linéaire n'allait pourtant pas de soi et n'est d'ailleurs pas entrée dans l'ordre de l'évidence. Il faut alors se demander ce qui a rendu possible cette opération de substitution et faire l'hypothèse selon laquelle elle repose peut-être sur un soubassement culturel peu aperçu mais qui pourrait expliquer comment ces objets techniques peuvent se charger d'une forte valeur imaginaire.

Sur le versant belge, il est certain que la plantation des talus s'est inscrite dans un projet de paysage explicite et porté par les pouvoirs publics. Bien que peu connu des Belges eux-mêmes, et bien que ses prescriptions aient été largement délaissées lorsque le royaume a commencé à fermer, à la faveur de son tournant libéral des années $1990^{22}$, les services qui avaient été chargés de la mise en œuvre de ce projet, ce Plan vert (Groenplan ${ }^{23}$. en néerlandais) a bel et bien été voulu par le ministère des Travaux publics et exposé en 1958 dans un livre diffusé dans tous les services d'infrastructures. Ce manifeste proposait que l'engagement des grands chantiers infrastructurels, notamment autoroutiers, occasionne un remodelage volontariste du paysage porteur de l'expression d'une identité nationale belge. Le Plan vert reposait sur une stratégie consistant à faire des infrastructures, reliant les grandes villes belges entre elles, le support même de l'identité nationale, ce choix surprenant étant induit par l'impossibilité, dans ce pays qui assemblait plusieurs capitales régionales de poids 
démographique équivalent, d'associer l'une d'entre elle, au détriment des autres, à cette expression nationale. Alors que l'image de la République française se superpose à celle de Paris, en Belgique l'équivalence des grandes villes ne pouvait être préservée qu'en concentrant l'effort de construction paysagère sur les infrastructures en ce qu'elles ne relèvent du territoire d'aucune de ces villes mais qu'elles les distribuent et les mettent en réseau toutes ensemble et dans un rapport d'absolue équivalence. Le Plan vert a montré comment ces voies devaient être plantées, jardinées, habitées d'œuvres d'art, architecturées au moyen de viaducs et de passerelles aux tracés élégants, et longées par des itinéraires piétonniers reconstituant les continuités de parcours que le tracé infrastructurel aurait interrompues (Delbaere, 2016, p. 77-78). Bien qu'ignoré, le Plan vert est symptomatique d'un statut donné jadis aux plantations de ces ouvrages, et explique peut-être pourquoi elles redeviennent aujourd'hui, au terme d'un débat parlementaire, une composante valorisée du paysage.

À l'inverse, l'absence d'un tel antécédent projectuel en France explique peut-être que l'évolution des mesures de gestion soit plus lente à s'engager. Dans l'imaginaire français, l'infrastructure est un pur objet technique, sans valeur culturelle, esthétique ou environnementale. Pourtant, dans le cas spécifique de la région Nord-Pas-de-Calais, les prémices d'une évolution de cette conception réifiante sont apparues dans le débat scientifico-politique depuis une vingtaine d'années et traversent régulièrement les débats que la région organise autour de ces sujets ${ }^{24}$.

De 1992 à 1998, en effet, la région Nord-Pas-de-Calais a été gouvernée par une alliance $\mathrm{du}$ parti socialiste et des Verts, lesquels en ont assuré la présidence pendant un mandat. Cette période a été marquée par l'engagement de grands projets à visée environnementale dont le point culminant a été la publication d'une "Trame verte et bleue » régionale assez pionnière, et dont la mise en œuvre est passée par un appel à volontariat des collectivités régionales et des acteurs de la société civile. Constatant que cet appel à projets restait dans une large mesure lettre morte, les instances régionales ont attribué cet échec au caractère trop abstrait du concept de trame verte et bleue, et ont choisi de lui substituer celui de forêt régionale ${ }^{25}$, selon une stratégie de reconfiguration conceptuelle déjà observée dans d'autres environnements régionaux (Cormier, 2011). En 2009, des objectifs de reforestation très ambitieux ont été fixés (180 000 hectares en 30 ans !) et un nouvel appel à projets a été lancé, mais celui-ci a rencontré l'écueil inverse: la forêt renvoie dans l'imaginaire de nos sociétés à un ensemble boisé de grande taille et massif, dont la plantation sur le parcellaire morcelé d'une région très marquée par l'urbanisation, l'industrie et l'openfield apparaît d'emblée impossible. Il a donc fallu tordre la notion de forêt et inventer ce que ses promoteurs ont appelé un nouveau modèle forestier, adapté à la complexité du territoire régional (Delbaere, 2009, p. 180). La forêt régionale ne serait pas un massif d'un seul tenant mais une constellation de petits bois de quelques hectares, reliés entre eux par des corridors boisés ou par des boisements en "pas japonais » encore plus petits.

28 Au même moment, la recherche en écologie du paysage débattait de la théorie «Single Large Or Several Small » (SLOSS), et conduisait un certain nombre de chercheurs (voir notamment Daniel et al., 2011) à montrer dans quelles conditions un boisement morcelé et linéarisé pouvait malgré tout représenter un gain significatif en biodiversité et rendre différents services écosystémiques (Clergeau, 2019, p. 48). La congruence de ces démarches diffuse tellement l'idée d'une reconfiguration de l'imaginaire forestier qu’à 
Lille un nouveau quartier se paraît du nom de «bois habité » (Delbaere, 2009, p. 176), ou que plus récemment au nord de Paris, le long du boulevard périphérique, on plantait une " forêt linéaire ». La plasticité du terme est peut-être aujourd'hui telle que le statut des talus boisés pourrait évoluer dans un sens analogue à celui de leurs voisins belges.

C'est ce que semble confirmer l'intérêt que la filière des métiers du bois dans les Hautsde-France manifeste pour l'invention d'une "sylviculture urbaine ", c'est-à-dire l'application aux boisements en milieu urbain de méthodes de gestion forestière, impliquant d'hybrider les visées économiques de l'exploitation du bois - orientée vers la valorisation en bois d'œuvre mais surtout en bois énergie - et celles de la protection et du développement de la biodiversité tout autant que des aménités paysagères. Fibois, l'association qui représente cette filière, a récemment organisé avec la Métropole européenne de Lille et la Maison des sciences de l'homme et de la société une journée d'étude consacrée au sujet, qui a permis de rassembler un public varié de chercheurs et d'enseignants, d'étudiants et de chargés de mission de collectivités territoriales et de services d'infrastructures. Les boisements de bord d'infrastructures y ont servi de cadre d'exploration du sujet ${ }^{26}$.

Figure 6. Carte des structures végétales du réseau d'infrastructures de l'Eurométropole Likoto

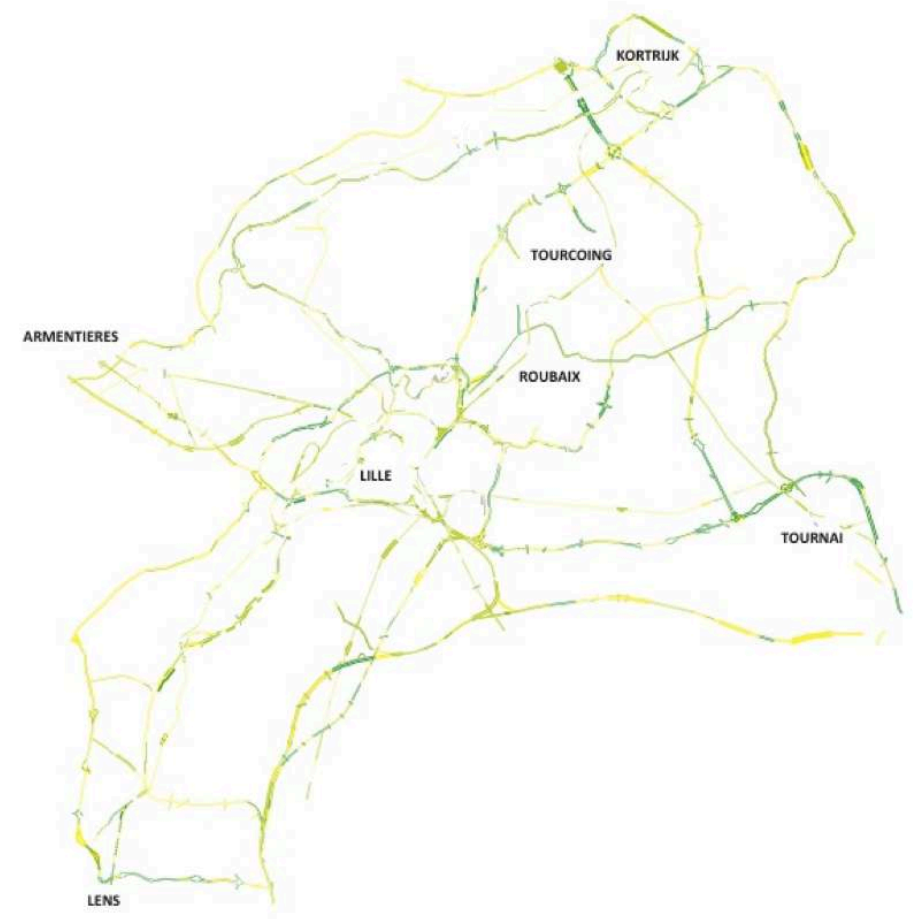

En jaune, les pelouses ; en hachuré vert, les fourrés et ensembles arbustifs ; en vert, les linéaires boisés.

Source : Rapport « En marge. Paysage et biodiversité des accotements infrastructurels de l'Eurométropole Likoto » (Pirve, 2015).

L'observation des différentes dynamiques que nous venons de décrire, et qui tendent peut-être aujourd'hui à faire émerger les boisements infrastructurels comme les 
armatures d'un paysage forestier d'un nouveau genre, documente un processus finalement assez rapide de fabrique du paysage. Il n'aura pas fallu 20 ans pour que ces marges inaperçues et à l'invisibilité notoire deviennent des éléments de cadre de vie parfois revendiqués, largement investis par la société civile jusqu'à y jouer un véritable rôle de refuge urbain, et relevant progressivement de mesures de gestion, et peut-être bientôt aussi d'aménagement, spécifiques. La rapidité du phénomène doit nous étonner alors que tant de projets de paysage peinent à voir le jour en suivant la procédure classique de production de l'espace public (programmation, conception, négociation, mise en œuvre, appropriation). Un tel paysage, si son émergence devait se confirmer, n'a été conçu par personne mais résulte de la congruence d'une multitude d'attentes et d'actions largement ignorantes les unes des autres. Il s'appuie sur une fondation culturelle ancrée dans des imaginaires peut-être lointains, autant que dans des archives projectuelles occupées par de grands projets d'aménagement du territoire oubliés mais bien réels. C'est pourquoi il est plus juste de désigner cette forme induite plutôt que produite de projet, dont l'instance d'émergence se situe moins sur la table à dessin des maitres d'œuvre que dans la démarche critique des chercheurs, par le terme de métaprojet (Delbaere, 2016 et 2020).

\section{BIBLIOGRAPHIE}

Ballard, J. G., L'île de béton (1974), Paris, Denoël, 2005.

Baud, L., « La gestion forestière des talus ferroviaires », dans « Un jardin linéaire et une trame verte pour l'Eurométropole? ", actes du séminaire organisé par la recherche En marge, Lille, MESHS, 4 avril 2012, p. 26-27.

Belhanafi, K.,Vandersmissen, R. et Dacquin, J., « La gestion des accotements autoroutiers de la région lilloise », 12 juin 2014, dans « Quelle gestion pour les accotements infrastructurels de l'Eurométropole Likoto?», actes de la journée d'étude, Villeneuve d'Ascq, ENSAPL, 28 octobre 2014, p. 51-52.

Bournonville, R. et Savaete, F., « La gestion des accotements des voiries départementales », 21 juin 2014, dans « Quelle gestion pour les accotements infrastructurels de l'Eurométropole Likoto ? ", actes de la journée d'étude, Villeneuve d'Ascq, ENSAPL, 28 octobre 2014, p. 49-50

Chelkhoff, G., Paris, M., Laroche, S. et Marchal, T., « La nature au bord de la route et de la voie ferrée. Des jardins collectifs pour une conception soutenable des infrastructures de transport terrestres », laboratoire Cresson, programme Ittecop, 2016.

Clergeau P., « De la théorie de la biogéographie insulaire (1963) à la conception actuelle des paysages urbains », Les Carnets du paysage, $n^{\circ}$ 35, Arles/Versailles, Actes Sud/ENSP, mai 2019, p. 48.

Collectif, « Un jardin linéaire et une trame verte pour l’Eurométropole ? », actes du séminaire organisé par la recherche En marge, Lille, MESHS, 4 avril 2012. 
Collectif, « Quelle gestion pour les accotements infrastructurels de l'Eurométropole Likoto ? ", actes de la journée d'étude, Villeneuve d'Ascq, ENSAPL, 28 octobre 2014

Cormier, L., « Les trames vertes : entre discours et matérialités, quelles réalités ? », thèse de géographie, université d'Angers, 2011

Daniel, H, Pellissier, V., Vallet, J. et Rozé, F., «La végétation des bois urbains », dans Clergeau, P. (dir.), Ville et Biodiversité. Les enseignements d'une recherche pluridisciplinaire, Rennes, Presses universitaires de Rennes, 2011.

Decourcelles, J.-P., dans « Un jardin linéaire et une trame verte pour l'Eurométropole ? ", actes du séminaire organisé par la recherche « En marge », Lille, MESHS, 4 avril 2012, p. 17.

Delbaere, D., « La forêt régionale : du mythe à la réalité territoriale, l'invention d'un nouveau modèle forestier? ", Les Cahiers thématiques, $\mathrm{n}^{\circ}$ 9, 2009.

Delbaere, D., Altérations paysagères. Huit projets pour une théorie critique du projet d'espace public, Marseille, Parenthèses, à paraître, 2020.

Delbaere, D., Table rase et Paysage. Une exploration des paysages de la modernité pour un renouveau critique du planisme, Paris, Éditions Pétra, 2016.

Delbaere, D et Ehrmann, S., « Des talus plein les yeux. De l'invisibilité notoire et de la prospection des espaces d'accotements », Projets de paysage, $n^{\circ} 13$, janvier 2016, URL : https:// www.projetsdepaysage.fr/fr/des_talus_pleins_les_yeux

Delbaere, D. (dir.), « En marge. Paysage et biodiversité des accotements infrastructurels de l'Eurométropole Lille-Kortrijk-Tournai », rapport de synthèse, ministère de l'Écologie, région Nord-Pas-de-Calais, conseil départemental du Nord, Métropole européenne de Lille, novembre 2015.

Lemoine, G., « Un diagnostic botanique “en marchant” peut-il apporter des données représentatives sur les enjeux relatifs à la flore et aux habitats des bordures des infrastructures de transport? » dans Delbaere, D. (dir.), « En Piste. Une randonnée scientifique pour une écologie intégrale des grandes infrastructures », programme de recherche Ittecop, novembre 2018.

Région Nord-Pas-de-Calais, « La forêt, des enjeux vitaux entre science et décision », colloque national, Lille, 23 novembre 2012.

Repoix, S. et Richard, H. (dir.), La Forêt au Moyen-Âge, Paris, Paris, Les Belles Lettres, 2019.

Spake, A., « Autoroute et paysage », Espaces verts, $n^{\circ}$ 14, avril/mai 1968, p. 8-25.

Spriet, Q., « La gestion des accotements des bords-à-canaux », 10 juin 2014, dans « Quelle gestion pour les accotements infrastructurels de l'Eurométropole Likoto?", actes de la journée d'étude, Villeneuve d'Ascq, ENSAPL, 28 octobre 2014, p. 53-54.

Vasset, P., Un livre blanc, Paris, Fayard, 2007.

\section{NOTES}

1. Deux programmes de recherche ont été concernés. «En marge. Paysage et biodiversité des accotements infrastructurels de l'Eurométropole Likoto" (Pirve, 2015) a regroupé les compétences de l'auteur, de Sabine Ehrmann (photographe), de Benoît Toussaint (botaniste), de Jean-Claude Bruneel (écologue), de Cédric Vanapelghem et Vincent Damoy (biologistes), de Francis Douay et Sébastien Détriché (pédologues), de Bruno Notteboom et Sam Lanckriet (architectes). «En piste, une méthode interdisciplinaire pour une écologie intégrale des bords 
d'infrastructure» (ITTECOP, 2018) a associé l'auteur à Sabine Ehrmann, Jean-Luc Brisson (artiste), Guillaume Lemoine (écologue),

Quentin Spriet (gestionnaire d'espaces naturels), Sébastien Nageleisen, Jean-Baptiste Litot et Nicolas Canova (géographes), Lucile Baud, Lucie Loosen et Elsa Grousseau (paysagistes).

2. Ministère de la Reconstruction et de l'Urbanisme, « Pour un Plan national d'aménagement du territoire ", 1950.

3. Selon Jean-Paul Decourcelles, secrétaire général aux affaires territoriales au sein de la direction régionale de la SNCF, dans «Un jardin linéaire et une trame verte pour l'Eurométropole?", actes du séminaire organisé par la recherche En marge, Lille, MESHS, 4 avril 2012, p. 17.

4. Entretien avec Karim Belhanafi, Raphaël Vandersmissen et Jean Dacquin, DIR Nord 12 Juin 2014, dans «Quelle gestion pour les accotements infrastructurels de l'Eurométropole Likoto?", actes de la journée d'étude, Villeneuve d'Ascq, ENSAPL, 28 octobre 2014, p. 51-52.

5. cf. note $\mathrm{n}^{\circ} 1$

6. Selon Jean-Paul Decourcelles, op.cit.

7. Selon Lucile Baud, ingénieure paysagiste à l'ONF, «La gestion forestière des talus ferroviaires ", dans «Un jardin linéaire et une trame verte pour l'Eurométropole? », op. cit., p. 26-27.

8. Lucile Baud, ingénieure paysagiste, a quitté cette fonction en 2018.

9. Selon Bertrand Duquesnoy, responsable «prospective et développement durable » au sein de la DIR Nord, dans « Un jardin linéaire et une trame verte pour l'Eurométropole ?», op. cit., p. 14.

10. Entretien avec Quentin Spriet, Espace naturel Lille Métropole, 10 juin 2014, dans "Quelle gestion pour les accotements infrastructurels de l'Eurométropole Likoto ?», op. cit., p. 53-54.

11. Entretien avec Rodrigue Bournonville et Franck Savaete, 21 juin 2014, dans «Quelle gestion pour les accotements infrastructurels de l'Eurométropole Likoto? », op. cit., p. 49-50.

12. Ibid., p. 49

13. Entretien avec Karim Belhanafi, Raphaël Vandersmissen et Jean Dacquin, op. cit., p. 52.

14. Entretiens avec les occupants de cabanes implantées à Lille, porte de Dunkerque, en bordure du canal de Deûle, octobre 2017.

15. Visite de l'aire d'accueil de Grenay (62) avec Marie-Paule Logie (AREAS Tsiganes et Voyageurs), 26 février 2016.

16. Entretien marchant avec Arnaud Lecouffe, conseil général du Nord, 21 juillet 2014.

17. Simon, C., Le Bois dont les rêves sont faits, Just Sayin' Films - Pio \& Co - Tipi'mages Productions, 2016.

18. Entretien avec Karim Belhanafi, Raphaël Vandersmissen et Jean Dacquin, op.cit., p. 52 ; entretien avec Quentin Spriet, op. cit., p. 53-54

19. Entretien avec Harold Grandjean, directeur de la Direction des études environnementales et paysagères, anciennement Direction des aménagements paysagers (DGO174) du Service public de Wallonie (SPW) Mobilité-Infrastructures, 30 juin 2014.

20. Entretien avec Harold Grandjean et Samantha Straet, 23 novembre 2018.

21. Cette équipe ne se compose pour l'instant que de Harold Grandjean (ingénieur horticole diplômé de l'Institut d'agronomie de Gembloux, et titulaire d'un certificat d'analyse de paysage), de Virginie Lefebure (paysagiste) et de Sophie Adam, Bertrand Lerot, Farah Roland et Samantha Straet, ingénieurs agronomes chargés de mission au sein de la Direction des études environnementales et paysagères dirigé par Harold Grandjean.

22. Entretien avec Michel Watillon, paysagiste au Plan vert retraité, 25 mars 2015.

23. Ministerie van Openbare Werken en van Wederopbouw, Het Groenplan, 1958.

24. Région Nord-Pas-de-Calais, colloque national «La forêt, des enjeux vitaux entre science et décision », Lille, 23 novembre 2012. 
25. Entretien avec Claudine Joalland, directrice de l'Environnement au conseil régional du NordPas-de-Calais, 27 janvier 2009.

26. Journée du 9 mars 2020. On trouvera les éléments présentés et discutés lors de cette journée grâce au lien suivant : https://www.bois-et-vous.fr/actualites/565-retours-sur-la-journee-foreturbaine-du-9-mars-2020

\section{RÉSUMÉS}

Les problèmes de gestion que posent les taillis boisés qui se sont formés par manque d'entretien sur les talus et les accotements plantés des grandes infrastructures de transport ouvrent la perspective d'un projet de paysage analysé ici à partir du cas de l'Eurométropole Likoto (LilleKortrijk-Tournai). Les gestionnaires d'infrastructures suivent une logique de contention de cette forêt linéaire qui se développe librement à distance des voies et des plateformes. Ces logiques d'abandon favorisent à leur tour des pratiques sociales d'habitats temporaires et de loisirs qui tirent parti du couvert «forestier ». Parallèlement, depuis quelques années, une approche plus raisonnée de ces boisements d'infrastructures, comme ressource pour leur valorisation esthétique mais aussi économique (production et exploitation de la biomasse), se fait jour. Le statut de ces boisements passe depuis quelques années de celui de contrainte à celui de ressource, y compris pour les instances qui en ont la charge. Une telle mutation des perceptions de cette forêt potentielle recueille peut-être l'héritage de projets de paysage déjà anciens, comme le Plan vert belge de 1958 ou la forêt régionale du Nord-Pas-de-Calais et le " nouveau modèle forestier » qu'elle a défendu. La plasticité du modèle forestier, motivée notamment par les débats théoriques de l'écologie du paysage (notamment la théorie des îles et le SLOSS), induirait de ce point de vue un réinvestissement des friches arborées des bords d'infrastructures comme d'une véritable forêt linéaire.

The problems posed in the management of the wooded coppices which form, due to a lack of maintenance, on the slopes of the embankments and in the planted areas along major transport infrastructures offer an opportunity to study the case of a landscape project in the Likoto Eurometropolis (Lille Kortrijk Tournai). The team in charge of managing the local transport infrastructures follow an approach based on the containment of this linear forest which has been allowed to develop freely at a distance from motorways, railway tracks and station platforms. Such approaches allowing vegetation to grow freely also encourage the social practices of temporary dwellings and leisure activities that take advantage of the "forest" cover. At the same time, in recent years has emerged a more reasoned approach to the afforestation of infrastructures as a resource for the aesthetic and economic development of these areas through the production and exploitation of the biomass. In recent years, even the authorities in charge of the maintenance of these transport infrastructures have come to see these forms of afforestation as a resource rather than a constraint. Such a change in the perception of this potential forest may be considered as the heritage of long-standing landscape projects such as the Belgian Green Plan of 1958 or the Nord-Pas-de-Calais regional forest and the "new forestry model" the latter has come to represent. The plasticity of the forest model, motivated by theoretical debates on landscape ecology (notably the island theory and the SLOSS), seen from this perspective, would imply a re-appropriation of these wooded wastelands in the form of a linear forest on the edges of transport infrastructures. 
INDEX

Mots-clés : infrastructures de transport, délaissés, sylviculture urbaine, trame verte urbaine, marges urbaines

Keywords : transport infrastructures, neglected areas, urban forestry, urban green belt, urban outskirts

\section{AUTEUR}

\section{DENIS DELBAERE}

Denis Delbaere est paysagiste concepteur et professeur « Ville et Territoire » à l'École nationale supérieure d'architecture et de paysage de Lille.

denisdelbaere59[at]gmail[dot]com 